\title{
Patrones generales de investigación en contabilidad: análisis de una muestra de universidades
}

\author{
General research patterns in accounting: analysis of a sample of \\ universities in Spain
}

José L. Arquero (arquero@us.es) ORCID: 0000-0002-7086-8812

Sergio M. Jiménez-Cardoso (smanuel@us.es) ORCID: 0000-0001-9583-4718

Joaquina Laffarga-Briones (quinal@us.es) ORCID: 0000-0002-3937-1242

Depto. de Contabilidad y Ec. Financiera, Universidad de Sevilla (España)

http://dx.doi.org/10.12795/EDUCADE.2018.i09.04

\begin{abstract}
RESUMEN: En España, las sucesivas reformas universitarias han tenido, entre sus objetivos potenciar la investigación universitaria, estableciendo requisitos de acceso y promoción cada vez más claramente ligados a la actividad investigadora y definiendo de forma más precisa (y estricta) qué se considera un output de investigación aceptable.
\end{abstract}

Con carácter general, la aplicación sistemática de estos criterios de evaluación ha llevado un incremento notable de la productividad científica en términos de presencia en bases de datos internacionales. Sin embargo, frente a estos efectos, positivos (mayor internacionalización, orientación a la investigación publicada en revistas de impacto), otros autores alertan de una caída de los resultados de investigación y del reconocimiento asociado en nuestra área.

En esta línea, el principal objetivo de este estudio es analizar el impacto de dichas políticas en la producción científica de los profesores de contabilidad en las universidades españolas tanto públicas como privadas.

Para ello se procede a determinar una muestra de universidades españolas, identificar a todos los profesores de contabilidad adscritos a dichas universidades y recopilar su producción científica en el período 2005-marzo de 2014 (en el que los criterios de evaluación ya están bastante definidos).

La conclusión principal que se obtiene de este estudio es que en el periodo considerado hay un conjunto relativamente elevado de profesores con una producción científica baja, y que la producción de trabajos considerados "de impacto" se concentra en un número relativamente reducido de profesores. Esta baja producción se concentra, para las públicas, en las categorías laborales con perfil no investigador y en los TEU y también es muy alta en los profesores de universidades privadas.

PALABRAS CLAVE: Investigación en contabilidad; evaluación de la investigación; incentivos.

\begin{abstract}
Recent University laws reforms in Spain sought to promote research by setting promotion and tenure criteria increasingly more related to research production and also by defining more specifically what could be considered acceptable outputs. In general terms, the systematic application of such criteria has caused an increase of research productivity in terms of presence in international databases and journals. However there are also some undesired effects, as some part of the academic staff could be reducing their research output and accounting research could be undervalued by new standards.
\end{abstract}

In this line the main aim of the present research is to investigate the effect of such policies on the accounting academic production in Spain in both public and private owned universities.

A sample of accounting university teachers was identified and the research opuput for those teachers included sought and analysed. The studied period covered 2005-2014, when new criteria were more internalized by academics. 
The main conclusion of our study is that the perceived improvement (in terms of high impact papers) is very concentrated on a small part of the population, whereas for the vast majority the effects have been the contrary: discourage for research. The vast majority of staff presents a low research production that is very acute on non-research contracts, tenured non-doctors (TEU) and private Universities teachers.

KEYWORDS: Accounting research. Assessment of research, incentives

Artículo. Recibido: 15-02-18 - Versión revisada: 20-04-18, Aceptado: 07-05-18

Licencia Creative Commons BY NC ND · 2018 • Asociación Española de Contabilidad y Administración de Empresas - AECA

\section{ANTECEDENTES}

En España, las sucesivas reformas universitarias, comenzando con la Ley Orgánica 11/1983, de 25 de agosto, de Reforma Universitaria (LRU) y la posterior Ley Orgánica 6/2001, de 21 de diciembre, de Universidades (LOU) han tenido, entre sus objetivos potenciar la investigación universitaria. Para ello, se crearon figuras contractuales específicamente orientadas a la investigación (con baja carga docente) y se crearon complementos salariales específicos por productividad investigadora (los conocidos sexenios, o tramos de investigación regulados inicialmente en el Real Decreto 1086/1989, de 28 de agosto, sobre retribuciones del profesorado universitario).

La obtención de estos tramos, inicialmente un mero complemento salarial no excesivamente generoso, ha ido creciendo en importancia, en la medida en que se ha constituido en un requisito para poder dirigir tesis doctorales, formar parte de comisiones, obtener proyectos y finalmente impactando en la carga docente de aquellos profesores que no han obtenido recientemente ese reconocimiento'. Por último, la relevancia de la investigación en este proceso de reforma queda patente en la institución de un sistema de evaluación previo ${ }^{2}$, como requisito para acceder a puestos docentes, en el que la actividad investigadora del candidato es determinante (ver los documentos de principios orientadores, ANECA, 2012).

Con carácter general, Jiménez-Contreras et al (2003) indican que la aplicación sistemática de estos criterios de evaluación, cada vez establecidos de una manera más clara, ha llevado un incremento notable de la productividad científica en términos de presencia en bases de datos internacionales. De esta manera, puede concluirse que esta política ha sido exitosa.

En nuestra área específica, el interés investigador se desarrolló más tarde que en otros campos. Considerada un área eminentemente técnica, dentro de las ciencias económicas y empresariales, la "investigación" contable centró tradicionalmente en la divulgación de la técnica, la resolución de problemas profesionales y en el análisis crítico y sistemático de las normas. Una revisión somera de los índices de las revistas de la década de los 80 evidencia el aspecto normativo-crítico de la investigación contable de la época (García-Benau y Laínez, 2004). En esta línea, Gonzalo y Mora (2010) resaltan que la reforma universitaria favoreció la presencia de profesores universitarios de contabilidad españoles en los foros y congresos internacionales, que

\footnotetext{
1 El Real Decreto-ley 14/2012, de 20 de abril, de medidas urgentes de racionalización del gasto público en el ámbito educativo modifica el Artículo 68 de la Ley Orgánica 6/2001, de 21 de diciembre, de Universidades, que trata de la dedicación del profesorado subiendo la carga docente a 32 créditos ECTS a quienes no hayan sometido a evaluación el primer período de seis años de actividad investigadora o que haya obtenido una evaluación negativa de dicho período.
}

2 Actualmente la acreditación: Ley Orgánica 4/2007, de 12 de abril y Real Decreto 1312/2007, de 5 de octubre. 
con el tiempo se hizo frecuente y también afectó al tipo de investigación, que comenzó a orientarse hacia trabajos más empíricos, en muchos casos buscando una proyección internacional. Los resultados del trabajo reciente de Arquero et al (2015) son claros en cuanto al impacto de estas políticas en el comportamiento investigador de los académicos de éxito, que se ha adaptado a la evolución de las exigencias de los sistemas de evaluación, internacionalizando las aportaciones y enfocándose, cada vez más claramente, a revistas académicas consideradas de impacto. En la misma línea, Moya et al (2015) indican como las presiones normativas orientan a los académicos a centrarse en los outputs más valorados (artículos académicos en determinado tipo de revistas).

Sin embargo, frente a estos efectos, positivos (mayor internacionalización, orientación a la investigación publicada en revistas de impacto), otros resultados alertan de una caída de los resultados de investigación en cuanto a volumen (Moya et al. 2015) o a reconocimiento: los resultados de investigación reconocidos en el área de la economía financiera y contabilidad siguen siendo en la actualidad los más bajos de todo el campo 8, lo que conlleva un sinfín de consecuencias negativas y es motivo de preocupación a todos los niveles (Cavero y Ferrández, 2013). Ciertamente, los indicadores de productividad de nuestra área parecen indicar que el esfuerzo investigador sigue siendo bajo.

El reciente trabajo de Moya et al. (2015) sugiere que los académicos jóvenes, con contratos no permanentes si orientan su investigación a la obtención de artículos considerados de alto impacto pero, para los profesores con contratos más estables, se observa una caída sustancial de la actividad investigadora.

En esta línea, el principal objetivo de este estudio es analizar la producción científica de los profesores de contabilidad en las universidades españolas tanto públicas como privadas.

\section{METODOLOGÍA}

A efectos de este estudio se consideran universidades españolas las que tienen su sede principal en España y profesores de contabilidad los que dedican la mayor parte de su docencia a las diferentes materias contables (fundamentos, normalización, estados contables, contabilidad y/o control de gestión o de costes, análisis contable, auditoría, contabilidad pública...).

La muestra de profesores se construyó eligiendo a todos los profesores de contabilidad que impartían docencia en una muestra estratificada de universidades españolas. El punto de partida es un listado de universidades españolas en las que se imparte docencia en contabilidad, considerando como tales aquellas que ofertan títulos de Grado en Administración y Dirección de Empresas (o similar) y/o Grado en Finanzas y Contabilidad (o similar). Esa información se obtuvo de la base de datos de Eroski y del mapa de universidades elaborado por el Ministerio de Educación corroborándola con la obtenida de las páginas institucionales e incluyendo a todas las instituciones (públicas, privadas, a distancia o presenciales) que ofertaran alguno de esos títulos, bajo cualquier variación de nombre.

La población de universidades se clasificó por tamaño, antigüedad y propiedad. Por tamaño se hicieron tres grupos: grandes (más de 20.000 alumnos), medianas (entre 20.000 y 5.000 alumnos) y pequeñas (menos de 5.000 alumnos). Por antigüedad se clasificaron en antiguas, fundadas hasta 1983 (inclusive), y nuevas, fundadas después de 1983. Se empleó como punto de corte el año de publicación de la Ley Orgánica 11/1983, de 25 de agosto, de Reforma Universitaria (LRU), en la creencia que esta norma podría suponer diferencias significativas en la estructura, objetivos y, por lo tanto, características de la producción científica universitaria. De todas formas, en 
1984 y 1985 no se produjo ninguna fundación, por lo que a efectos prácticos, los grupos de universidades están formadas antiguas (fundadas hasta 1983) y nuevas (fundadas desde 1986). Por propiedad se clasificaron en públicas y privadas. Se consideran públicas las universidades que constituyen una administración pública. Se consideran privadas el resto, aunque sean propiedad (parcial o total) de organismos 0 consorcios o dependan directa o indirectamente de administraciones públicas.

La clasificación anterior evidenció que prácticamente todas las universidades grandes son públicas y antiguas. Casi todas las pequeñas son privadas y nuevas. La mayor dispersión se da entre las universidades medianas, de las que hay públicas y privadas y antiguas y nuevas. Por ello, se optó por estratificar la muestra en 6 grupos. Las universidades se clasificaron en estos grupos, eligiéndose aleatoriamente lo que se considera una muestra representativa de cada estrato. A continuación se indican los tipos de universidades y la muestra seleccionada:

- Universidades grandes (3 de 23)

- Universidades pequeñas (4 de 27)

- $\quad$ Universidades medianas públicas antiguas (2 de 8)

- Universidades medianas públicas nuevas (2 de 16)

- Universidades medianas privadas antiguas (2 de 5)

- Universidades medianas privadas nuevas (2 de 4)

Puede observarse que la muestra no es proporcional, siendo el muestreo más intenso, en las poblaciones más pequeñas. Por eso ha sido preciso contar con un mayor número relativo de universidades privadas dado el menor tamaño que tienen en cuanto número de profesores. Finalmente, las universidades que han conformado la muestra han sido 7 públicas y 8 privadas. Sus características se presentan en la tabla 1.

Tabla 1. Características de la muestra de universidades

\begin{tabular}{|c|c|c|c|c|}
\hline \multirow[b]{2}{*}{ Universidades Públicas } & \multicolumn{2}{|c|}{ Fecha de creación } & \multicolumn{2}{|c|}{ Tamaño ( $n^{\circ}$ de alumnos) } \\
\hline & Antes de 1985 & Tras 1985 & $5.000-20.000$ & Más de 20.000 \\
\hline Alicante & $x$ & & & $X$ \\
\hline Gerona & & $x$ & $x$ & \\
\hline León & 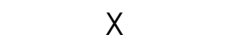 & & $x$ & \\
\hline Miguel Hernández-Elche & & $x$ & $x$ & \\
\hline Murcia & $x$ & & & $x$ \\
\hline Oviedo & $x$ & & $x$ & \\
\hline Valladolid & $X$ & & & $X$ \\
\hline Universidades Privadas & Antes de 1985 & Tras 1985 & $\begin{array}{l}\text { Menos de } \\
5.000\end{array}$ & $5.000-20.000$ \\
\hline Alfonso X & & $\mathrm{X}$ & & $\bar{X}$ \\
\hline Católica de Valencia & $x$ & & & $x$ \\
\hline Católica S. Antonio de Murcia & & $x$ & & $x$ \\
\hline Europea M. de Cervantes & & $x$ & $x$ & \\
\hline Internac. de Cataluña & & $x$ & $x$ & \\
\hline Navarra & $x$ & & & $x$ \\
\hline Nebrija & & $x$ & $x$ & \\
\hline San Jorge & & $x$ & $x$ & \\
\hline
\end{tabular}

Una vez seleccionada la muestra de universidades se identificó al profesorado de contabilidad de cada una de ellas. La selección se realizó empleando, principalmente, las listas de profesores por departamento disponibles en las páginas web de las universidades. Cuando existía un departamento de contabilidad con docencia única en el área, todos los profesores del departamento fueron calificados como profesores de contabilidad. Cuando el departamento incluía otras disciplinas pero existía un área de contabilidad, todos los profesores del área de contabilidad fueron calificados como profesores de contabilidad. En la mayoría de las ocasiones, sin 
embargo, las listas de profesores más concretas disponibles hacían referencia a departamentos o áreas de Economía Financiera o Economía de la Empresa. En los casos en los que las páginas web de las universidades no permitían segregar a los profesores de contabilidad, se identificó las asignaturas impartidas por cada profesor del departamento o área en la que estaban incluidas las materias contables. Se calificaron como profesores de contabilidad aquellos que impartían única 0 principalmente materias contables. Cuando la información disponible en la página web de las universidades no permitía conocer las materias impartidas por algunos profesores, o la dedicación relativa a cada una de ellas (si impartían materias contables y no contables), se contactó con el departamento. La información proporcionada por estas fuentes posibilitó reconocer a los profesores de contabilidad. Este mecanismo se empleó en un número significativamente pequeño de casos.

Para verificar la fiabilidad de las listas incluidas en las páginas web de las universidades se empleó la lista de profesores pertenecientes a ASEPUC. Pudo comprobarse que la representatividad de ASEPUC varía mucho dependiendo de la universidad. El listado de ASEPUC permitió identificar sólo a algunos (muy pocos) profesores no incluidos en las páginas web de las universidades. Ello se debe a que, al menos en algunas universidades, los listados publicados en sus páginas web no incluyen becarios 0 profesores con estancias temporales en otras universidades. Dado el resultado de la confrontación, consideramos bastante fiable y exhaustiva la lista de profesores empleada en la investigación.

Parece frecuente que, en las universidades pequeñas, los profesores no se agrupen en departamentos (o al menos no parecen existir departamentos con presencia en las páginas web de las universidades). En estos casos, los profesores de contabilidad fueron identificados buscando los profesores responsables de la docencia de las asignaturas contables de los estudios en administración y dirección de empresas (las universidades pequeñas no parecen ofrecer un GFICO o similar).

La muestra total está compuesta por 207 profesores, 169 de universidades públicas y 38 de privadas (tabla 2). Prácticamente el $40 \%$ de los profesores de contabilidad de las universidades privadas son profesores de la Universidad de Navarra (15 de 38).

Tabla 2. Número de profesores de la muestra por universidad

\begin{tabular}{l|cc}
\hline Universidad & Privadas & Públicas \\
\hline Alicante & & 32 \\
Girona & & 12 \\
León & & 15 \\
Miguel Hernández-Elche & & 15 \\
Murcia & & 30 \\
Oviedo & & 39 \\
Valladolid & 2 & 26 \\
Alfonso X & 6 & \\
Católica de Valencia & 3 & \\
Católica San Antonio de Murcia & 1 & \\
Europea Miguel de Cervantes & 4 & \\
Internacional de Catalunya & 15 & \\
Navarra & 4 & \\
Nebrija & 3 & \\
San Jorge & & \\
Total & $\mathbf{3 8}$ & $\mathbf{1 6 9}$ \\
\hline
\end{tabular}

De la muestra de profesores de contabilidad se obtuvieron los siguientes datos:

- Nombre y apellidos

- Correo electrónico

- Universidad 
$\begin{array}{ll}\text { - } & \text { Departamento (si existe) } \\ \text { Categoría }\end{array}$

La categoría fue fácil de obtener en las universidades públicas. Las universidades privadas no suelen tener una jerarquía similar. Al comprobar la coincidencia entre las categorías publicadas en las páginas web de las universidades y las de ASEPUC pudo observarse que los datos contenidos en las páginas web de las universidades son más actuales y concretos.

Identificado el profesorado que constituye la muestra, se recopiló su producción científica (aportaciones) en el período 2005-marzo de 2014. Para efectuar esta tarea se han consultado las siguientes bases de datos y buscadores:

\author{
- $\quad A B I$ Inform \\ - Google Scholar \\ - Dialnet (básicamente para los trabajos en español)
}

También se empleó la información que proporcionan las páginas web de las universidades sobre su producción científica.

Frecuentemente, las bases de datos anglosajonas registran los nombres de origen español de forma diversa, incompleta y/o defectuosa. Por ello, en la búsqueda de las aportaciones se han empleado las variaciones más típicas o esperables del nombre o apellidos de los autores. Por ejemplo, un profesor de contabilidad llamado López García, P. se habría buscado también como Garcia, P.L. Lopez-Garcia, P y Lopez, P. Posteriormente los resultados obtenidos por cada autor se han filtrado y tratado de forma manual.

En esta parte del estudio se ha considerado cada profesor como un caso. Por tanto, las variables no son cada aportación individual, sino el número y tipo de aportaciones de cada profesor. El criterio básico de clasificación de las aportaciones es su tipo (artículo, contribución a libro, contribución a congreso). Entre los artículos se han distinguido los no indexados de los indexados (se han considerado indexados los publicados en revistas incluidas en las bases del SSCI-JCR (en adelante JCR) o de SCIMAGO-SCOPUS (en adelante Scopus). Para determinar si una revista estaba indexada en el JCR o en Scopus hemos empleado los listados de correspondientes a los trabajos publicados año 2012 (último disponible al realizar esta tarea). Las revistas que están incluidas en los dos listados (Scopus y JCR) se han asignado al que recibe mayor valoración (JCR).

También se ha distinguido entre los artículos publicados en revistas contables o en revistas de otras áreas (se han considerado revistas de contabilidad aquellas en cuyo nombre aparece el término "Contabilidad", "Accounting" o alguno similar o que haga referencia directa a cuestiones contables o de auditoría (por ejemplo, Abacus o Auditing).

\title{
3. RESULTADOS
}

Comenzaremos analizando la producción publicada como artículos de revista, debido a que generalmente se le concede más importancia que a otro tipo de aportaciones para evaluar la producción científica de los profesores universitarios.

La distribución y recorrido del número de artículos publicados por cada profesor de la muestra durante el período de estudio se muestra en el gráfico 1. Esta variable tiene un recorrido entre "ninguno" y "40". Dada su distribución, la media (de valor 3,76 y con una desviación típica de 6,7) carece de representatividad. La moda tiene un valor "0" y la mediana se sitúa en un único trabajo publicado en revistas. 
Dado que el periodo cubierto es de, aproximadamente, 10 años, hemos reclasificado el número de artículos en tres intervalos: "ninguno", "de 1 a 10" y "más de 10". Los resultados presentados en el gráfico 2 indican que un $45 \%$ de la muestra no parece haber publicado ningún artículo (o al menos no hemos podido identificar ninguno en nuestra búsqueda), un $44 \%$ ha publicado menos de un trabajo de media por año y sólo un $11 \%$ más de un trabajo de media por año.

Gráfico 1. Distribución del número de artículos publicados por cada profesor de contabilidad durante el período de estudio (2005-T12014)

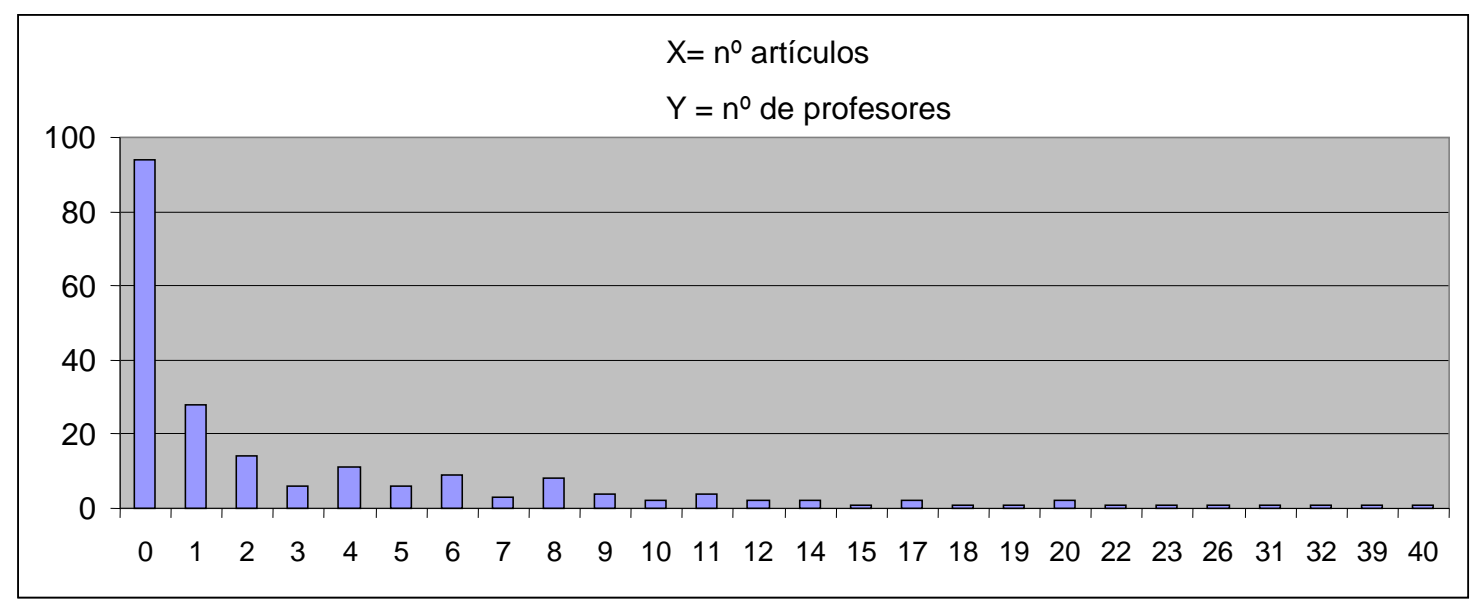

Gráfico 2. Distribución (por intervalo) del número de artículos publicados por profesores de contabilidad durante el período de estudio (2005-T12014)

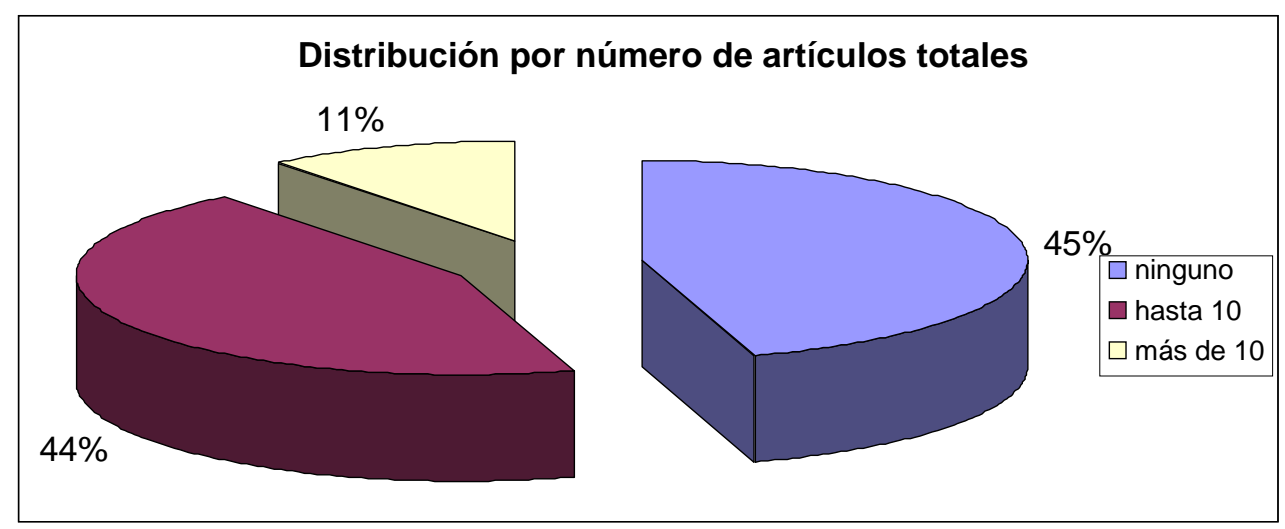

La importancia creciente que se está otorgando a los trabajos publicados en revistas indexadas justifica que realicemos un análisis detallado de las contribuciones de este tipo. El gráfico 3 muestra el recorrido y la distribución del número de artículos publicados en revistas incluidas en el JCR por cada profesor. Esta variable tiene un recorrido de "cero" a "19". La gran mayoría de profesores analizados (165 de 207) no han publicado ningún artículo en revistas indexadas en el JCR y 18 profesores sólo tienen uno. 
Gráfico 3. Distribución del número de artículos, indexados en el JCR, publicados por profesores de contabilidad durante el período de estudio (2005-T12014)

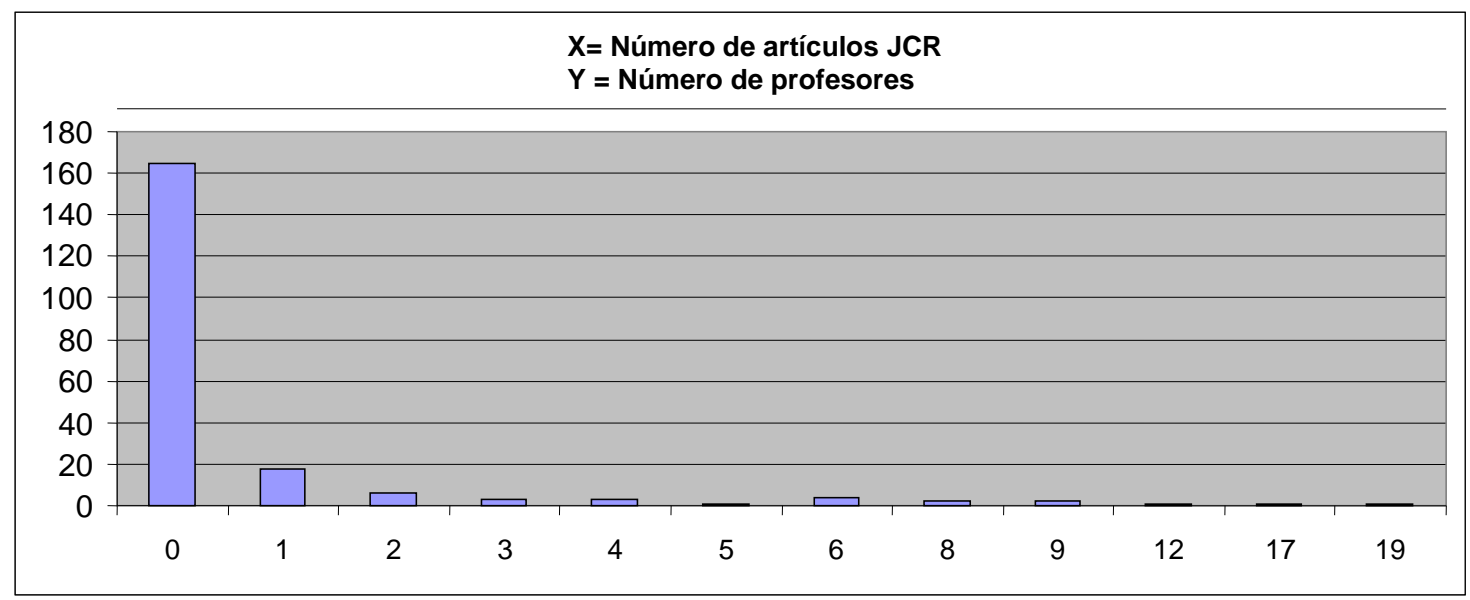

Si reclasificamos el número de artículos publicados en las revistas JCR en: "0", "de 1 a 5" y "más de 5", puede observarse (en el gráfico 4) que el $80 \%$ de los profesores de contabilidad de la muestra tienen un valor 0 , sólo el 15\% "de 1 hasta 5" y únicamente un $5 \%$ tiene "más de 5" trabajos publicados en revistas indexadas en el JCR. Hemos optado por este criterio de reclasificación porque permite identificar a aquellos profesores que, durante el período de estudio (casi diez años), consiguen al menos 5 aportaciones que permitirían una evaluación positiva de un sexenio.

Gráfico 4. Distribución (por intervalo) del número de artículos, indexados en el JCR, publicados por profesores de contabilidad durante el período de estudio (2005-T12014)

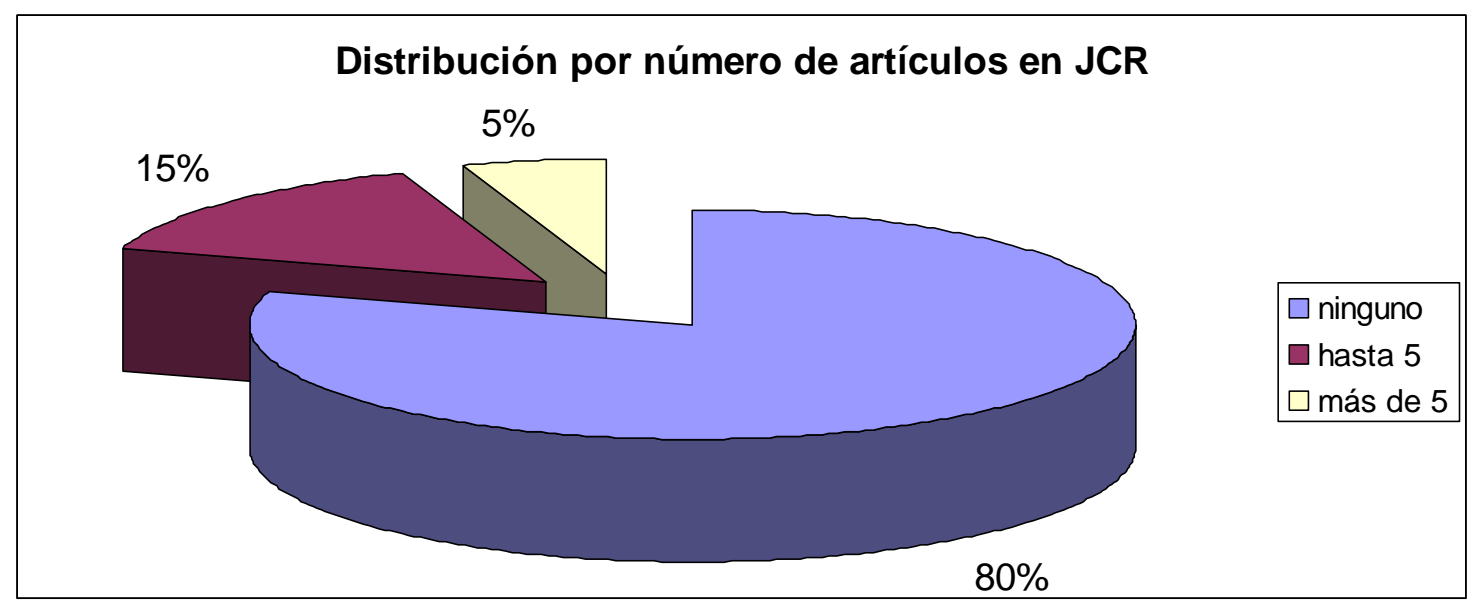

Tras el JCR, Scopus se usa frecuentemente como indicador de calidad de los artículos publicados en revistas. El gráfico 5 muestra el recorrido y distribución del número de artículos publicados en revistas indexadas en Scopus. En la muestra, esta variable tiene un recorrido más corto, entre cero y seis, lo que no es coherente con el mayor número de revistas académicas cubierto por esta base de datos en comparación con la cobertura del SSCI-JCR.

Al igual que ocurría con los artículos publicados en las revistas indexadas en el JCR, la mayoría de los profesores de la muestra no tiene ningún artículo publicado en revistas indexadas en Scopus durante el período de estudio. Si reclasificamos los valores, usando el criterio empleado anteriormente, puede observarse que el $81 \%$ de los profesores universitarios de contabilidad de la muestra no tiene ningún trabajo 
publicado en una revista incluida en este listado, un $18 \%$ de 1 a 5 trabajos y sólo un $1 \%$ más de 5 trabajos (ver gráfico 6).

Gráfico 5. Distribución del número de artículos, indexados en Scopus, publicados por profesores de contabilidad durante el período de estudio (2005-T12014)

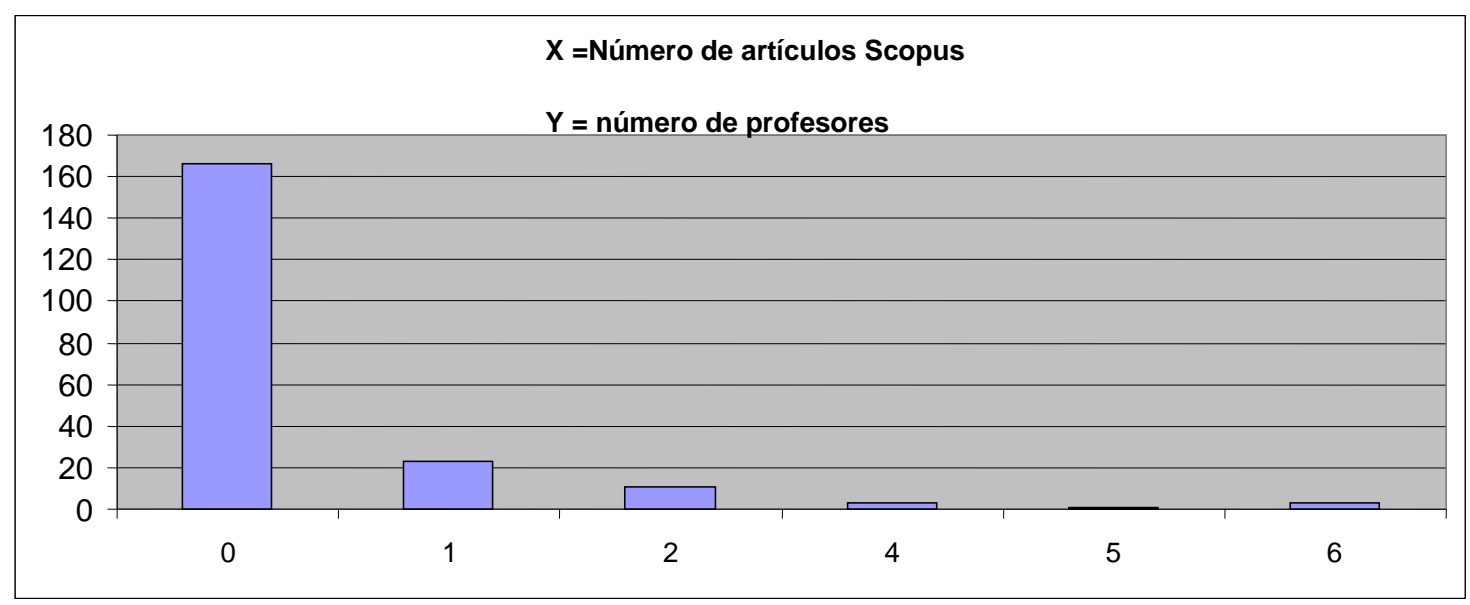

Gráfico 6. Distribución (por intervalo) del número de artículos, indexados en Scopus, publicados por profesores de contabilidad durante el período de estudio (2005-T12014)

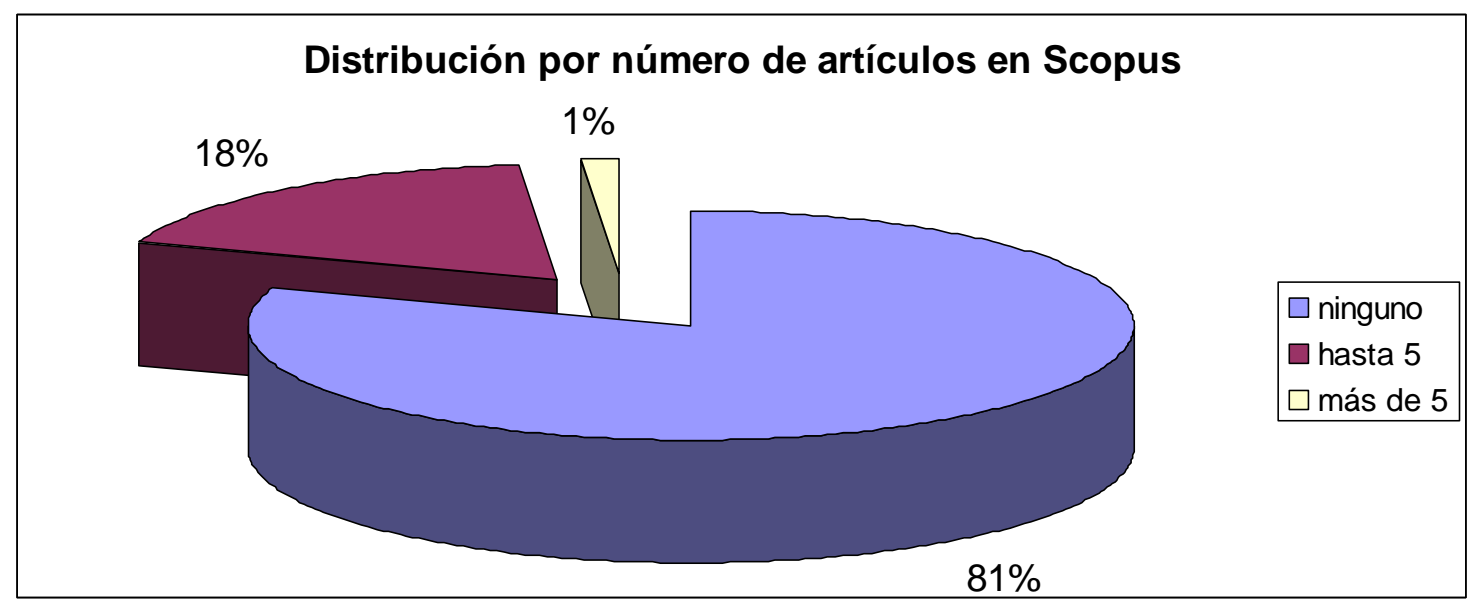

Si analizamos conjuntamente las aportaciones en revistas indexadas (ver gráfico 7), podemos observar que un $72 \%$ de la muestra no tiene ningún trabajo publicado en una revista indexada en el JCR o en Scopus, un $21 \%$ tiene entre 1 y 5 trabajos y sólo un $7 \%$ más de 5 . De estos datos se deduce que, aunque hay profesores que tienen artículos publicados en revistas indexadas en Scopus y no en JCR y viceversa, la mayoría de los que publican en revistas indexadas en JCR también lo hacen en revistas indexadas en Scopus (y viceversa). Al $45 \%$ de profesores que no publican artículos en el período analizado (ver gráfico 2), se suma otro $27 \%$ que no publica artículos en revistas indexadas (para llegar hasta el $72 \%$ que figura en el gráfico 7). El resto de los artículos corresponden, obviamente, a los publicados en revistas no indexadas. El gráfico 8 describe el recorrido y frecuencia de esta variable. Es destacable que 98 profesores (un 47,3\% de la muestra) no publican artículos en revistas no indexadas (al $45 \%$ de profesores que no han publicado artículos se une un pequeño grupo de profesores que sólo publican en revistas indexadas). 
Gráfico 7. Distribución (por intervalo) del número de artículos indexados publicados por profesores de contabilidad durante el período de estudio (2005-T12014)

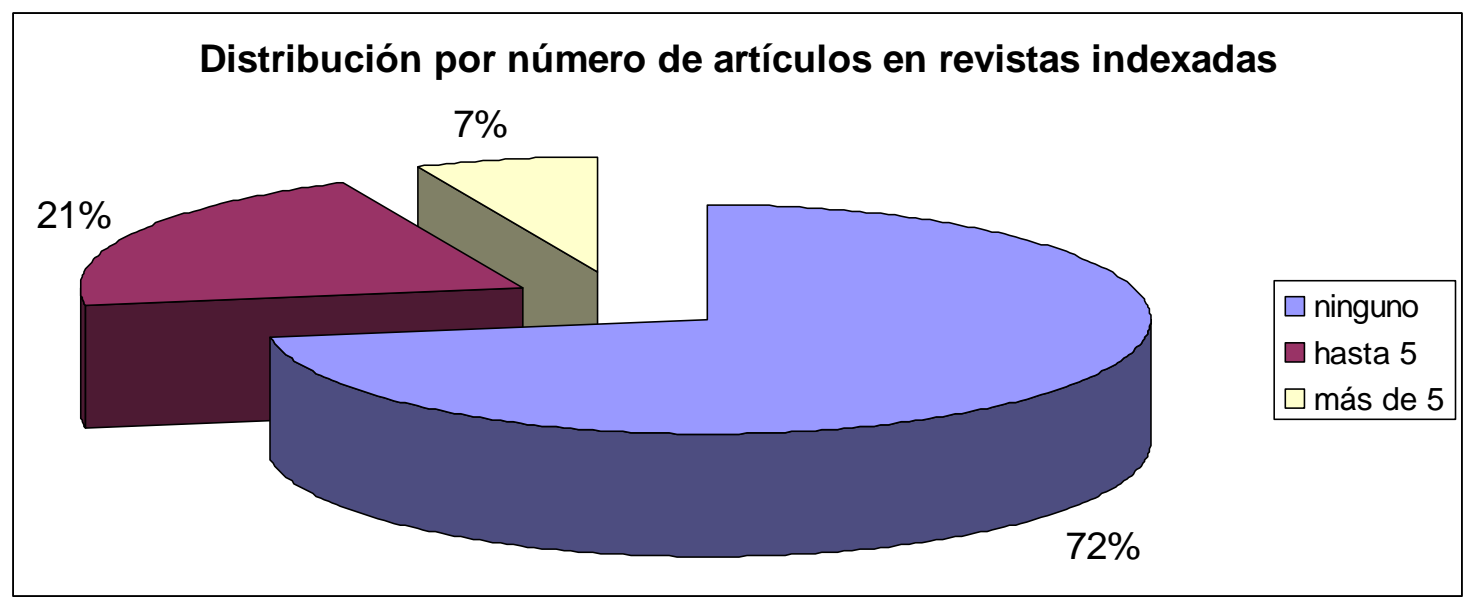

Gráfico 8. Distribución del número de artículos no indexados publicados por profesores de contabilidad durante el período de estudio (2005-T12014)

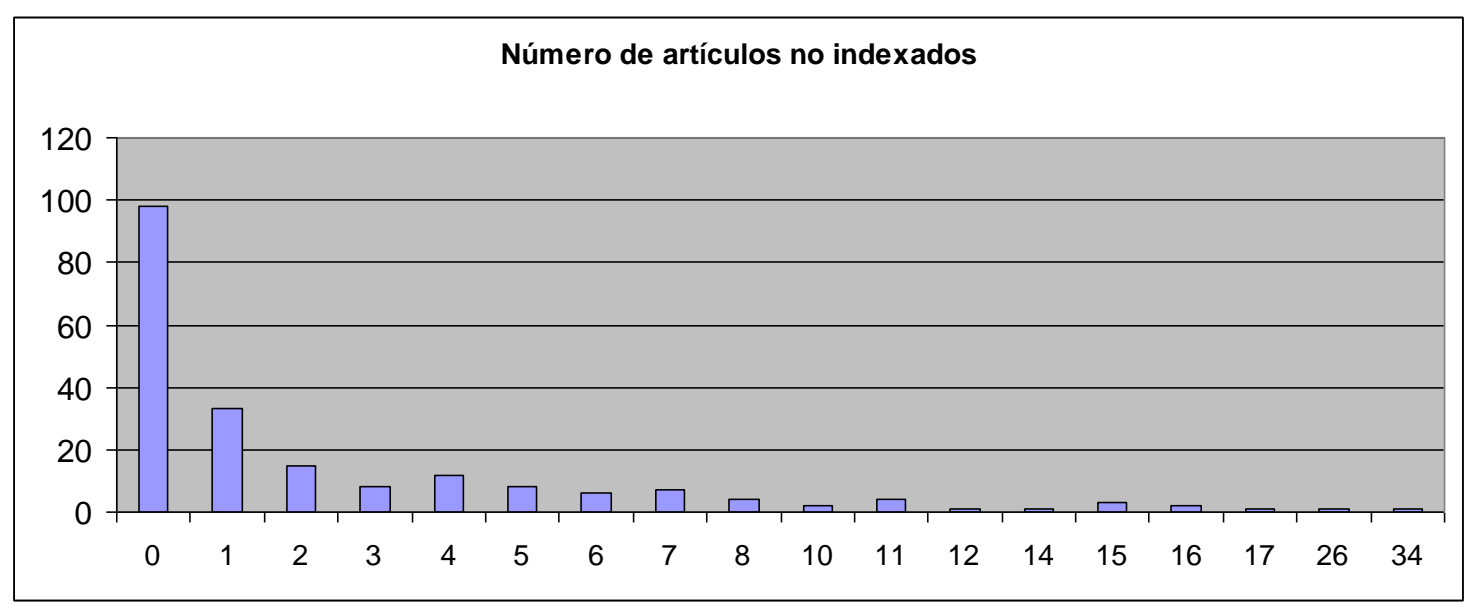

En el análisis previo se han incluido todos los artículos publicados en revistas, con independencia de si la revista en la que se publicaba era contable o no. Ahora distinguiremos entre los artículos publicados en revistas de contabilidad o en revistas de otras áreas. Recordemos que, a efectos de este estudio, hemos calificado como revistas de contabilidad aquellas en cuyo título aparece el término "contabilidad" "accounting", algún término relativo a la contabilidad (v.g. auditoría), o están específicamente en listados del área (v.g. Abacus).

El gráfico 9 presenta la distribución de los autores según el porcentaje de trabajos indexados que publican en revistas de contabilidad. Es una distribución bimodal, que acumula gran parte de los casos en los extremos. Es decir, que la mayoría de los autores: a) publican todos sus artículos indexados en revistas contables, o b) no publican ninguno de sus trabajos en revistas contables. El gráfico 10 acumula la distribución anterior en varios intervalos. En él puede observarse que un $28 \%$ de los profesores de contabilidad no publican sus mejores trabajos en revistas de contabilidad y otro $16 \%$ menos de un tercio. 
Gráfico 9. Distribución del porcentaje de artículos indexados publicados en revistas contables por profesores de contabilidad durante el período de estudio (2005-T12014)

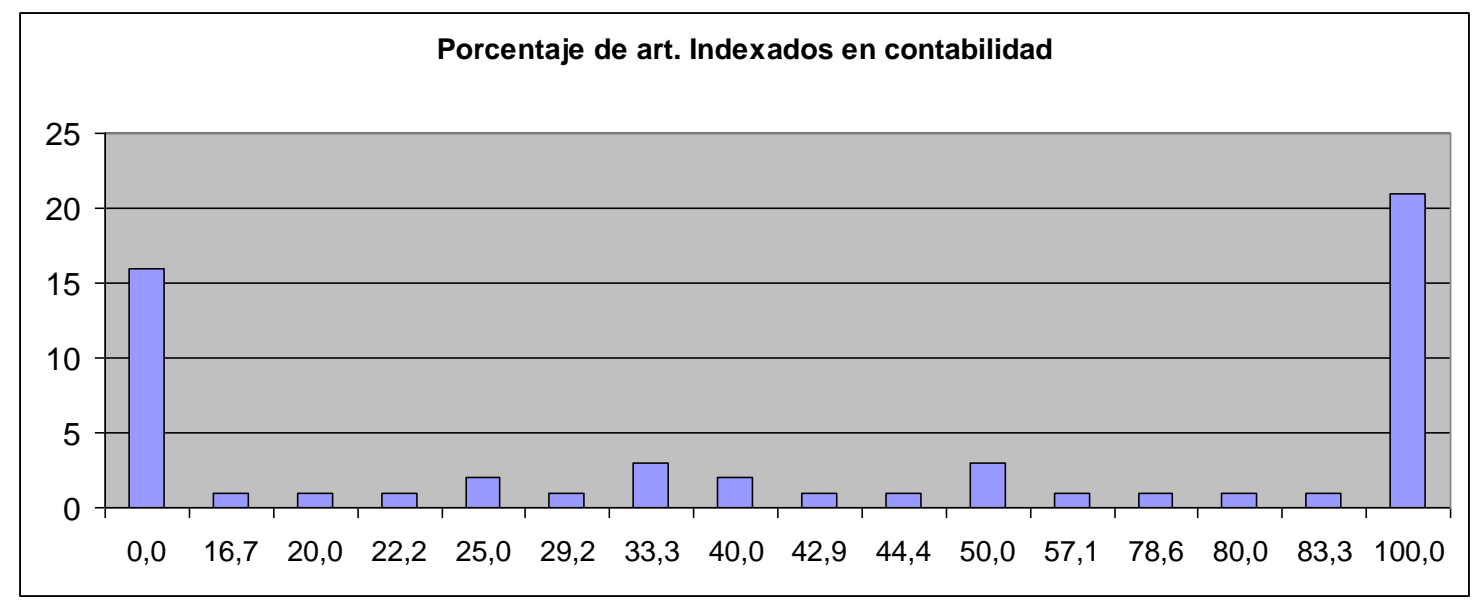

Gráfico 10. Distribución (por intervalo) del porcentaje de artículos indexados publicados en revistas contables por profesores de contabilidad durante el período de estudio (2005-T12014)

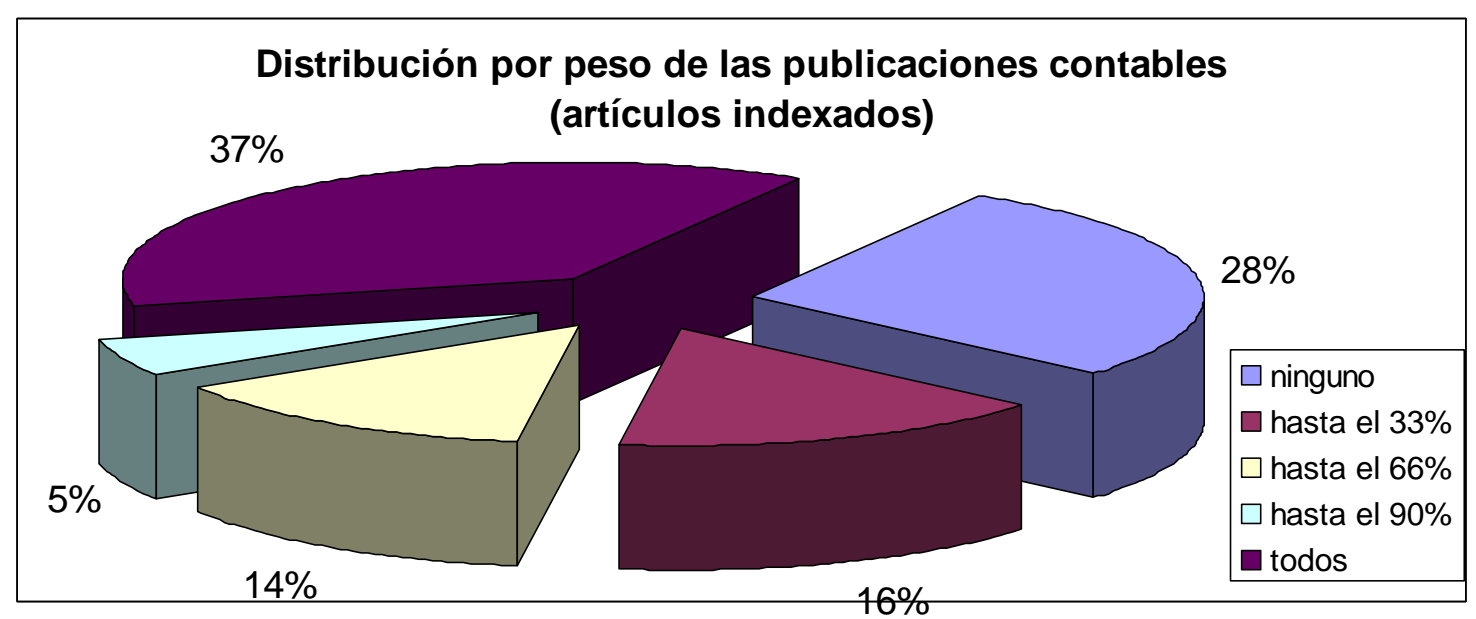

Ciertamente, la polarización del porcentaje se debe a que muchos profesores de contabilidad han publicado un número de artículos indexados muy reducido (o un único artículo) en el período de estudio. Por ello, los escasos artículos publicados se polarizan en revistas contables o no contables. Para evitar este problema, el gráfico 11 limita la muestra a los profesores de contabilidad que tienen 5 o más trabajos publicados en revistas indexadas. En este subgrupo de profesores de contabilidad (los más prolíficos), un $22 \%$ publica todos sus trabajos en revistas de contabilidad y un $56 \%$ publica menos de la mitad de sus artículos en revistas de contabilidad.

La proporción de artículos publicados en revistas contables puede estar influida por varios factores. Se ha argumentado que la escasez de revistas contables indexadas en el JCR o Scopus (o clasificadas en cuartiles bajos) motiva a los profesores de contabilidad a publicar sus trabajos en revistas de interés más amplio (por ejemplo, Business o Management) que acepten artículos dedicados a la contabilidad, aunque en su cabecera no aparezca una referencia explícita a ella. Por similares motivos, los profesores de contabilidad que desarrollan su investigación en áreas limítrofes con otras, podrían preferir publicar sus artículos en revistas de estas otras áreas, sobre todo si están mejor clasificadas. Tampoco puede descartarse que, con el objetivo de conseguir publicaciones de alto impacto, algunos profesores de contabilidad estén optando por investigar en áreas no contables. La tabla 3 proporciona información 
sobre la extensión de este fenómeno. Puede observarse que aproximadamente entre un 50 y un $60 \%$ de los artículos escritos por profesores de contabilidad se publican en revistas no contables. Este intervalo parece algo más amplío en el caso de los profesores de universidades privadas (del 37 al 77\%). Puede observarse que este fenómeno no se limita a los mejores trabajos, siendo aún más frecuente en la categoría de los artículos publicados en revistas "no indexadas".

Gráfico 11. Distribución (por intervalo) del porcentaje de artículos indexados publicados en revistas contables por profesores de contabilidad que durante el período de estudio (2005T12014) han publicado 5 o más artículos en revistas indexadas

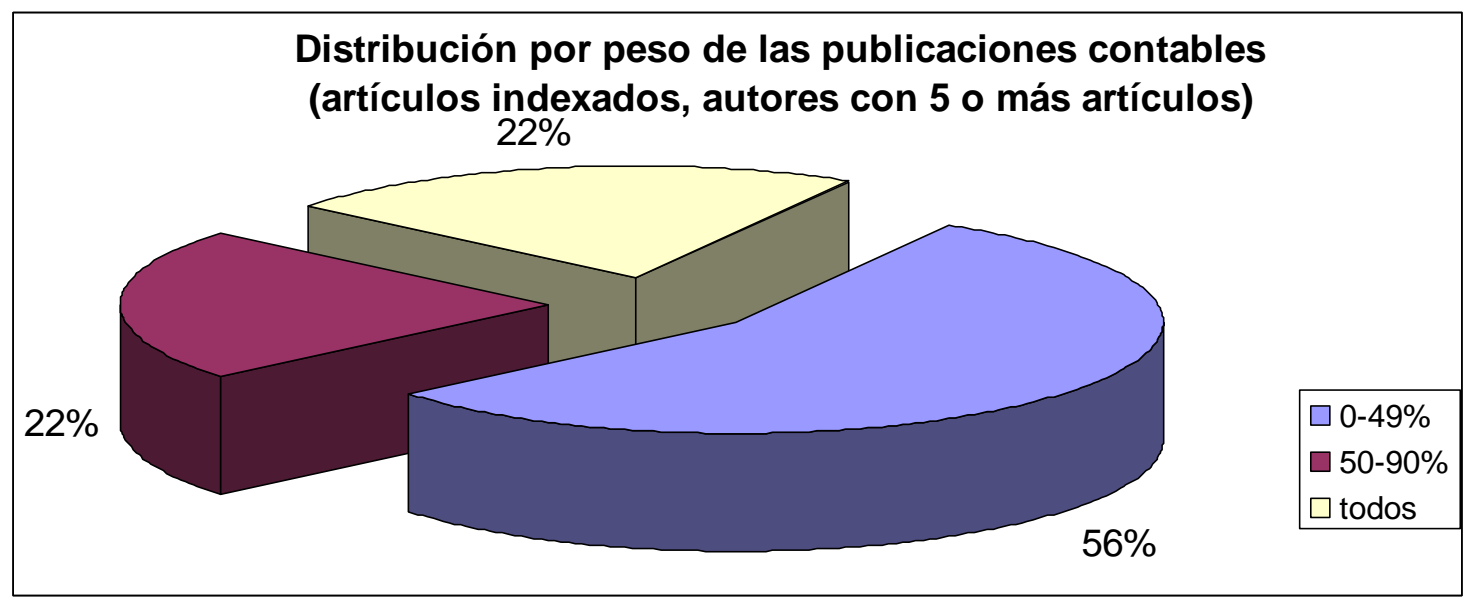

Tabla 3. Distribución de la producción de artículos por tipo de revista y tipo de contrato durante el período de estudio (2005-T12014)

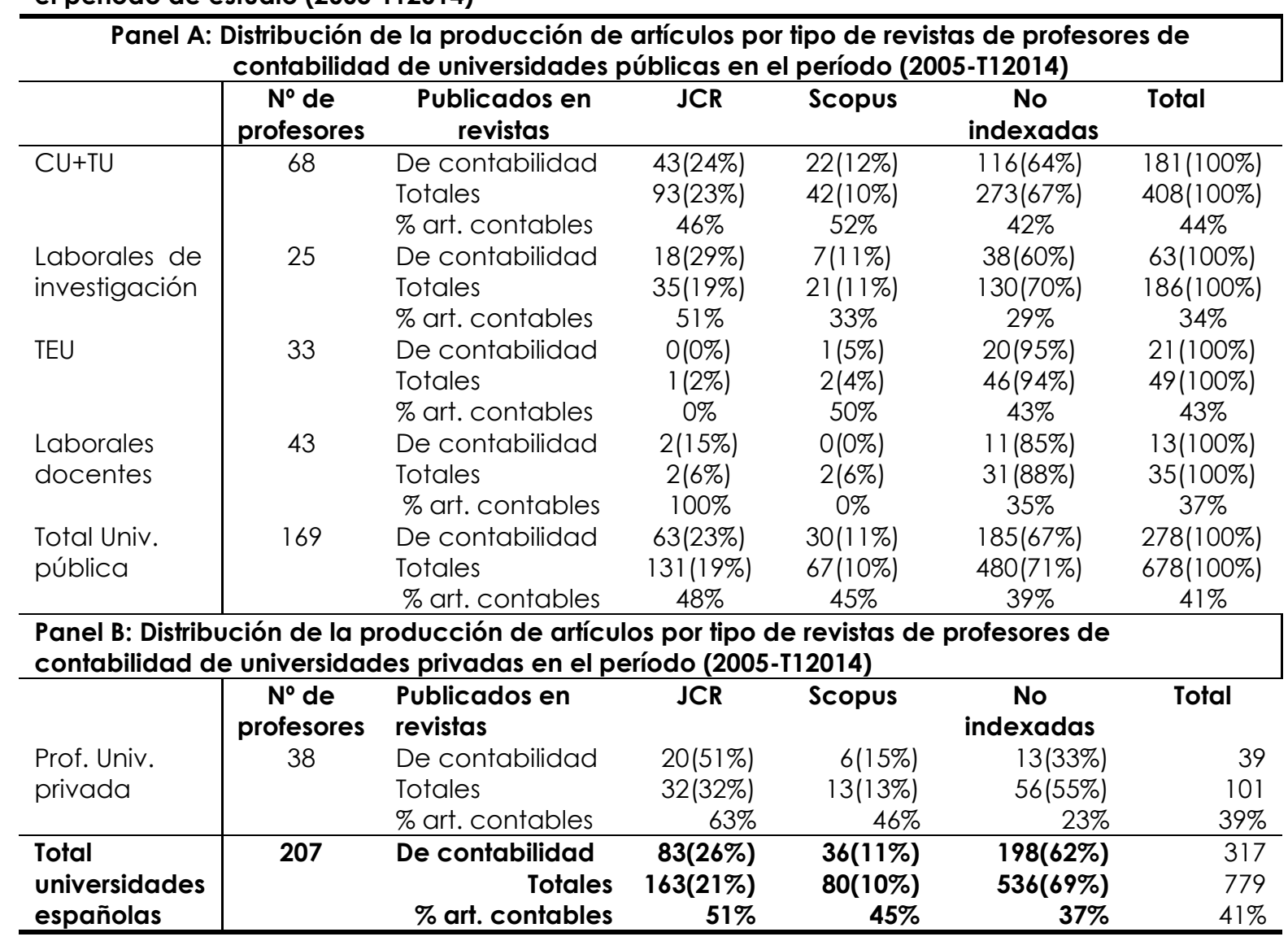


Una revisión de las revistas no contables en las que publican los profesores de contabilidad son coherentes con las hipótesis apuntadas anteriormente. Las revistas no contables más frecuentemente elegidas por los profesores de contabilidad son de management o business, aunque no son infrecuentes las de finanzas, economía o administración pública. Sólo una minoría de trabajos se publica en revistas con una conexión más distante, como ingeniería, física o transporte.

Como hemos indicado anteriormente, una parte significativa de la muestra parece no haber publicado durante el período de estudio. Esto es especialmente cierto cuando nos referimos a los artículos publicados en revistas indexadas. La tabla 4 describe los artículos indexados publicados por los 7 profesores de contabilidad más prolíficos de la muestra, distinguiendo entre aquellos que más publican en revistas indexadas en el JCR y los que lo hacen en revistas indexadas en Scopus (aunque mayoritariamente son los mismos autores).

Tabla 4. Producción de artículos publicados en revistas indexadas en JCR y SCOPUS de los 7 profesores más prolíficos durante el período de estudio (2005-T12014)

\begin{tabular}{lccc}
\hline \multicolumn{2}{c}{ Panel A: Profesores más prolíficos en la publicación de JCR en el período de estudio (2005-T12014) } \\
\hline Profesor & Universidad & $\begin{array}{c}\text { Artículos JCR } \\
\text { Total }\end{array}$ & $\begin{array}{c}\text { Artículos JCR (n-\%) } \\
\text { revistas contables }\end{array}$ \\
\hline 1019 TU & Pública A & 19 & $4-21 \%$ \\
1018 TU & Pública A & 17 & $3-18 \%$ \\
1095 TUP & Privada B & 12 & $6-50 \%$ \\
1027 TU & Pública A & 9 & $4-44 \%$ \\
1147 TU & Pública C & 9 & $2-22 \%$ \\
1091 PUP & Privada B & 8 & $7-88 \%$ \\
1008 AD & Pública A & 8 & $1-13 \%$ \\
\multicolumn{4}{c}{} \\
\% sobre producción total de la muestra & 82 & $27-33 \%$ \\
& Total muestra & 163 & $83-51 \%$ \\
\end{tabular}

\begin{tabular}{lccc}
\hline \multicolumn{4}{c}{ Panel B: Profesores más prolíficos en la publicación de SCOPUS en el período de estudio (2005-T12014) } \\
\hline Profesor & Universidad & $\begin{array}{c}\text { Artículos } \\
\text { Scopus (total) }\end{array}$ & $\begin{array}{c}\text { Artículos Scopus (n-\%) } \\
\text { revistas contables }\end{array}$ \\
\hline 1095 TUP & & 6 & $2-33 \%$ \\
$1027 \mathrm{TU}$ & Privada B & 6 & $2-33 \%$ \\
$1091 \mathrm{PUP}$ & Pública A & 6 & $4-67 \%$ \\
$1019 \mathrm{TU}$ & Privada B & 5 & $3-60 \%$ \\
$1018 \mathrm{TU}$ & Pública A & 4 & $4-100 \%$ \\
$1024 \mathrm{TU}$ & Pública A & 4 & $3-75 \%$ \\
$1009 \mathrm{CD}$ & Pública A & 4 & $1-25 \%$ \\
& Pública A & 35 & $19-54 \%$ \\
& Total & 80 & $36-45 \%$ \\
\% sobre producción total de la muestra & $44 \%$ & $53 \%$ \\
\hline
\end{tabular}

TU: titular de universidad, PUP: profesor de universidad privada, TUP: titular de universidad privada, AD: ayudante doctor. CD: contratado doctor

Puede observarse que estos profesores suponen casi el $50 \%$ de la producción en revistas indexadas de la muestra (constituida por 207 profesores). La proporción de artículos que cada uno de ellos publica en revistas contables indexadas en Scopus es similar a la muestra general (54 vs. $45 \%$ ). Por el contrario, la proporción de artículos que publican en revistas contables indexadas en el JCR es mucho menor a la media (33 vs $51 \%$ ) y a las que publican en revistas indexadas en Scopus. Por tanto, los autores que publican más artículos de calidad lo hacen, fundamentalmente, en revistas no contables en 2 de cada 3 ocasiones. Estos datos son coincidentes con lo apuntado por Arquero. Jiménez-Cardoso y Laffarga (2017), quienes analizando los patrones de investigación de los profesores acreditados encontraron una alta concentración de 
trabajos en revistas no contables. Es destacable asimismo, que entre los autores más prolíficos no hay ninguno que, al menos a la fecha de obtención de los datos, fuese catedrático encontrándose, además una fuerte concentración en una universidad pública y en una privada de la muestra, cuya orientación investigadora es muy diferente al resto de las universidades de su categoría.

El gráfico 12 describe las aportaciones de los profesores de contabilidad en forma de participaciones en libros o congresos. Como en casos anteriores, puede observarse que una parte relevante de la muestra no realizó ninguna aportación de este tipo en el período de estudio. Es posible que los profesores universitarios estén poco motivados a redactar capítulos de libros. Si esta producción tiene un fin docente, generalmente son inútiles para promocionar $u$ obtener algún incentivo. Aún en el caso de que el destino de los capítulos de un libro sea divulgar literatura científica, generalmente su puntuación como mérito suele ser significativamente más baja que la de un artículo publicado en una revista indexada en el JCR. También es probable que las aportaciones que han tomado la forma de libro y, sobre todo, las que han tomado la forma de capítulo de libro sean más difíciles de identificar, siendo posible que una parte significativa de las aportaciones que toman esta forma no hayan sido identificadas en esta investigación. La tabla 5 incluye una segmentación de las aportaciones en forma de libro o capítulo de libro por tipo de contrato. Aunque se observan algunas diferencias, éstas no son significativas estadísticamente.

Gráfico 12. Distribución (por intervalo) de participaciones en libros y congresos de profesores de contabilidad durante el período de estudio (2005-T12014)

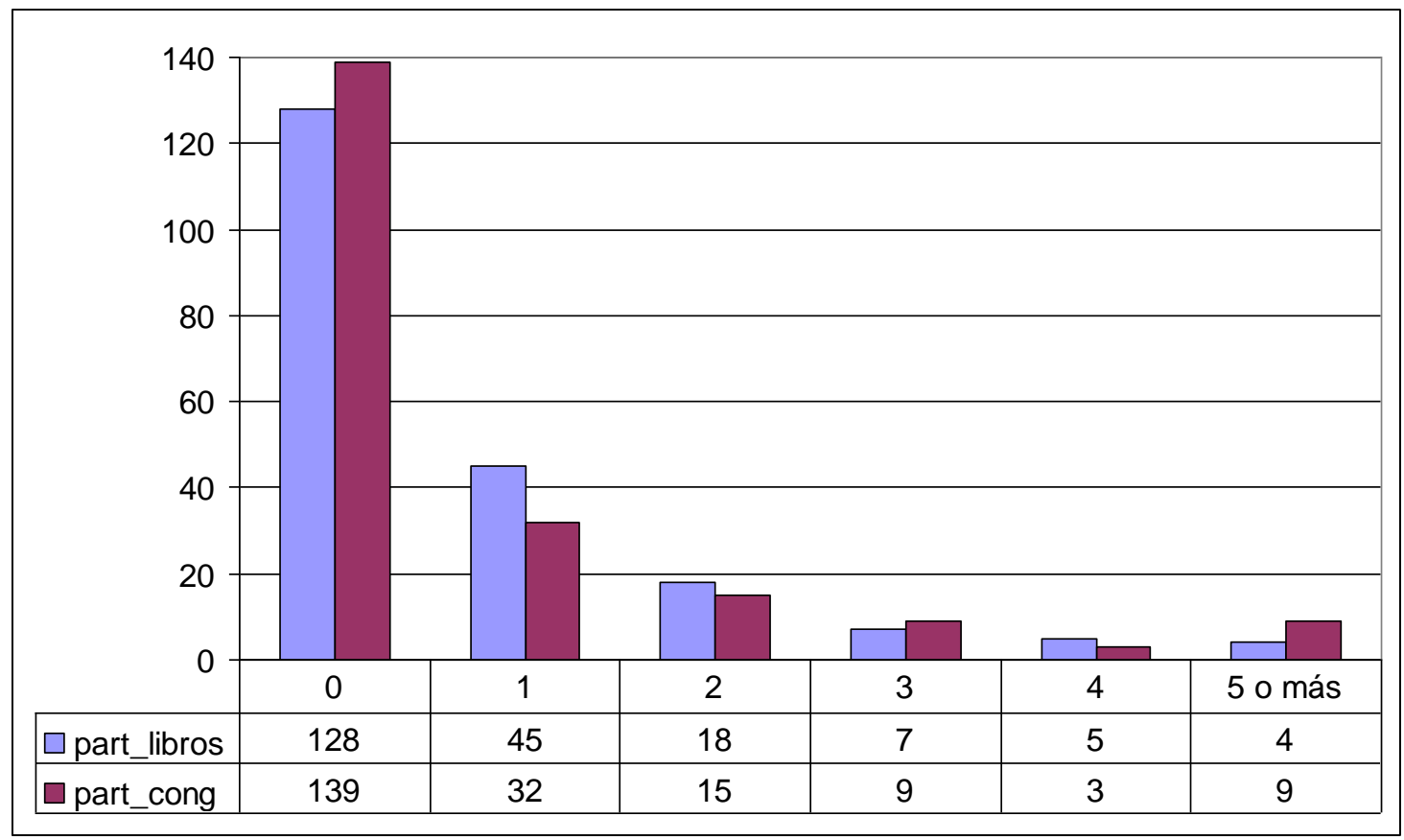

Respecto a las aportaciones a congresos, hemos detectado que su cobertura por las bases de datos, y por tanto su visibilidad, es muy reducida. El número de aportaciones identificadas en nuestra investigación es, en nuestra opinión, muy inferior al real, por lo que extraer conclusiones sobre estos datos puede ser arriesgado. Las causas que podrían explicar esta baja visibilidad son las siguientes:

- Sólo aparecen los congresos que han sido recogidos por Dialnet o muy correctamente identificados en otro tipo de base de datos. 
- La mayoría de los congresos relevantes en nuestra área fallan al identificar en sus actas en formato electrónico el congreso de que se trata, por lo que no son reconocidos como tales. La falta de identificadores (metadatos) para los buscadores aumenta este problema.

- Algunos otros (por ejemplo, los congresos de la EAA) no permiten el acceso en abierto ni siquiera a los libros de resúmenes, por lo que su visibilidad deviene en casi nula.

- Casi ninguna universidad y muy pocos autores tienen páginas mínimamente exhaustivas de su producción científica (en el mejor de los casos se indican las publicaciones más relevantes, en la mayoría, nada).

A esto se une el problema, ya señalado anteriormente, de la identificación de autores por buscadores diseñados para formatos de nombre anglosajones, que da lugar a múltiples identificaciones posibles para un solo autor.

Tabla 5. Participaciones en libros por tipo de contrato durante el período de estudio (2005T12014). Porcentaje sobre total de profesores por tipo de contrato

\begin{tabular}{clcccc}
\hline Participaciones en libros & \multicolumn{3}{c}{ Tipo de contrato* } & Total \\
\cline { 3 - 4 } & Funcionario & $\begin{array}{c}\text { Laboral de } \\
\text { investigación }\end{array}$ & $\begin{array}{c}\text { Laboral } \\
\text { docente }\end{array}$ & \\
\hline 0 & Recuento & 53 & 18 & 57 & 128 \\
& \% por tipo de contrato & $52,5 \%$ & $64,3 \%$ & $73,1 \%$ & $61,8 \%$ \\
1 & Recuento & 27 & $5 * *$ & 13 & 45 \\
& \% por tipo de contrato & $26,7 \%$ & $17,9 \%$ & $16,7 \%$ & $21,7 \%$ \\
2 & Recuento & 13 & 2 & 3 & 18 \\
& \% por tipo de contrato & $12,9 \%$ & $7,1 \%$ & $3,8 \%$ & $8,7 \%$ \\
30 & Recuento & 8 & 3 & 5 & 16 \\
más & \% por tipo de contrato & $7,9 \%$ & $10,7 \%$ & $6,4 \%$ & $7,7 \%$ \\
\hline
\end{tabular}

* Son funcionarios los Catedráticos y titulares, tanto de universidad como de escuela. Son laborales de investigación los profesores contratados, ayudantes y becarios. Es personal laboral docente los profesores asociados y colaboradores.

** 5 profesores del grupo de personal investigador tienen un libro o capítulo de libro en el período 2005-

T12014 (un 17,9\% de los profesores incluidos en el grupo de personal laboral de investigación).

No se observan diferencias significativas en la estructura de producción entre universidades públicas y privadas, ni en general (ver tabla 6), ni respecto a los artículos indexados (ver tabla 7). Por el contrario, si se aprecia una diferencia significativa (al $1 \%$ ) en la estructura de la producción entre las distintas categorías de profesores, tanto en general (ver tabla 8) como en los artículos indexados (ver tabla 9). De esta forma, catedráticos, titulares de universidad y contratados laborales de perfil investigador (frente a titulares de escuela universitaria y contratados laborales de perfil docente) muestran una mayor propensión a publicar. Los profesores de universidad privada muestran una posición intermedia entre estos dos grupos. La diferencia de categorías podría explicar diferencias entre la producción entre las universidades fundadas antes y después de la aprobación de la LRU (ver tabla 10), así como la diferencia entre la producción de universidades pequeñas, medianas y grandes (ver tabla 11), ya que unas y otras parecen tener estructuras de personal significativamente distintas (al $0,1 \%)$. Esta diferencia de estructura de la producción se observa en el conjunto de artículos, libros y capítulos de libros (ver tabla 12), que se muestra significativa al $5 \%$, pero no respecto a los artículos indexados (ver tabla 13). Por número de alumnos, las diferencias en la estructura de producción son estadísticamente insignificantes respecto a la producción general (tabla 14) y de artículos indexados (tabla 15). 
Tabla 6. $\mathrm{N}^{\circ}$ de profesores por tipo de universidad e intervalo de producción (artículos, libros y capítulos de libro) durante el período de estudio (2005-T12014). Porcentaje sobre total de profesores por tipo de universidad

\begin{tabular}{|c|c|c|c|c|}
\hline \multicolumn{2}{|c|}{ Artículos, libros y capítulos de libros } & \multicolumn{2}{|c|}{ Tipo de universidad } & \multirow[t]{2}{*}{ Total } \\
\hline & & Privada & Pública & \\
\hline \multirow[t]{2}{*}{ Ninguno } & Recuento & 16 & 58 & 74 \\
\hline & $\%$ por tipo & $42,1 \%$ & $34,3 \%$ & $35,7 \%$ \\
\hline \multirow[t]{2}{*}{1 a 10} & Recuento & $19^{*}$ & 91 & 110 \\
\hline & $\%$ por tipo & $50,0 \%$ & $53,8 \%$ & $53,1 \%$ \\
\hline \multirow[t]{2}{*}{ Más de 10} & Recuento & 3 & 20 & 23 \\
\hline & $\%$ por tipo & $7,9 \%$ & $11,8 \%$ & $11,1 \%$ \\
\hline Total & & 38 & 169 & 207 \\
\hline
\end{tabular}

* 19 profesores de universidad privada tienen una producción de artículos, libros y capítulos de libro entre 1 y 10 en el período 2005-2014 (un 50\% de los profesores de universidad privada de la muestra).

Tabla 7. $\mathrm{N}^{\circ}$ de profesores por tipo de universidad e intervalo de producción (artículos indexados) durante el período de estudio (2005-T12014). Porcentaje sobre total de profesores por tipo de universidad

\begin{tabular}{|c|c|c|c|c|}
\hline \multicolumn{2}{|c|}{ Artículos indexados } & \multicolumn{2}{|c|}{ Tipo de universidad } & \multirow[t]{2}{*}{ Total } \\
\hline & & Privada & Pública & \\
\hline \multirow[t]{2}{*}{ Ninguno } & Recuento & 30 & 120 & 150 \\
\hline & $\%$ por tipo & $78,9 \%$ & $71,0 \%$ & $72,5 \%$ \\
\hline \multirow[t]{2}{*}{1 a 5} & Recuento & $5^{*}$ & 38 & 43 \\
\hline & $\%$ por tipo & $13,2 \%$ & $22,5 \%$ & $20,8 \%$ \\
\hline \multirow[t]{2}{*}{ Más de 5} & Recuento & 3 & 11 & 14 \\
\hline & $\%$ por tipo & $7,9 \%$ & $6,5 \%$ & $6,8 \%$ \\
\hline Total & Recuento & 38 & 169 & 207 \\
\hline
\end{tabular}

* 5 profesores de universidad privada tienen una producción de artículos indexados entre 1 y 5 en el período 2005-2014 (un 13,2\% de los profesores de universidad privada de la muestra).

Tabla 8. $\mathrm{N}^{\circ}$ de profesores por tipo de contrato e intervalo de producción (artículos, libros y participaciones en libros) durante el período de estudio (2005-T12014). Porcentaje sobre total de profesores por tipo de contrato

\begin{tabular}{llrrrr}
\hline Tipo de contrato* & & \multicolumn{3}{c}{$\begin{array}{c}\text { Artículos, libros y } \\
\text { participaciones en libros }\end{array}$} & \multirow{2}{*}{ Total } \\
\cline { 2 - 4 } & & Ninguno & $\mathbf{1}$ a 10 & más de 10 & \\
\hline CU+TU & Recuento & 8 & 48 & 12 & 68 \\
& \% de tipo de contrato & $11,8 \%$ & $70,6 \%$ & $17,6 \%$ & $100,0 \%$ \\
Laboral de & Recuento & 6 & $16 * *$ & 6 & 28 \\
investigación & \% de tipo de contrato & $21,4 \%$ & $57,1 \%$ & $21,4 \%$ & $100,0 \%$ \\
TEU & Recuento & 16 & 16 & 1 & 33 \\
& \% de tipo de contrato & $48,5 \%$ & $48,5 \%$ & $3,0 \%$ & $100,0 \%$ \\
Laboral docente & Recuento & 35 & 18 & 1 & 54 \\
Universidad & \% de tipo de contrato & $64,8 \%$ & $33,3 \%$ & $1,9 \%$ & $100,0 \%$ \\
privada & Recuento & 9 & 12 & 3 & 24 \\
Total & \% de tipo de contrato & $37,5 \%$ & $50,0 \%$ & $12,5 \%$ & $100,0 \%$ \\
\hline
\end{tabular}

*Se ha considerado laboral de investigación a profesores contratados, ayudantes y becarios. Se incluyen en el personal laboral docente a profesores asociados y colaboradores.

** 16 profesores de contabilidad con contrato laboral de investigación tienen una producción de artículos, libros y participaciones en libros entre 1 y 10 en el período 2005-2014 (un 57,1\% de la muestra de laborales de investigación). 
Tabla 9. $N^{\circ}$ de profesores por tipo de contrato e intervalo de producción (artículos indexados) durante el período de estudio (2005-T12014). Porcentaje sobre total de profesores por tipo de contrato

\begin{tabular}{llrrrr}
\hline Tipo de contrato* & & \multicolumn{3}{c}{ Artículos indexados } & Total \\
\cline { 3 - 4 } & & Ninguno & $\mathbf{1}$ a 5 & más de 5 & \\
\hline CU+TU & Recuento & 40 & 20 & 8 & 68 \\
& \% de tipo de contrato & $58,8 \%$ & $29,4 \%$ & $11,8 \%$ & $100,0 \%$ \\
Laboral de & Recuento & 13 & $12 * *$ & 3 & 28 \\
investigación & \% de tipo de contrato & $46,4 \%$ & $42,9 \%$ & $10,7 \%$ & $100,0 \%$ \\
TEU & Recuento & 30 & 3 & 0 & 33 \\
& \% de tipo de contrato & $90,9 \%$ & $9,1 \%$ &, $0 \%$ & $100,0 \%$ \\
Laboral docente & Recuento & 50 & 4 & 0 & 54 \\
Universidad & \% de tipo de contrato & $92,6 \%$ & $7,4 \%$ &, $0 \%$ & $100,0 \%$ \\
privada & Recuento & 17 & 4 & 3 & 24 \\
Total & \% de tipo de contrato & $70,8 \%$ & $16,7 \%$ & $12,5 \%$ & $100,0 \%$ \\
\hline
\end{tabular}

* Se ha considerado laboral de investigación a profesores contratados, ayudantes y becarios. Se incluyen en el personal laboral docente a profesores asociados y colaboradores.

** 12 profesores de contabilidad con contrato laboral de investigación tienen una producción de artículos indexados entre 1 y 5 en el período 2005-2014 (un 42,9\% de la muestra de laborales de investigación).

Tabla 10. Estructura del profesorado por tipo de contrato y antigüedad de la universidad durante el período de estudio (2005-T12014)

\begin{tabular}{llrrr}
\hline Tipo de contrato & \multicolumn{2}{c}{ Fundación de la Universidad } & \multirow{2}{*}{ Total } \\
\cline { 2 - 4 } & & Antes de $\mathbf{1 9 8 5}$ & Después de 1985 & \\
\hline CU+TU & Recuento & 62 & 6 & 68 \\
& \% sobre universidad & $38,0 \%$ & $13,6 \%$ & $32,9 \%$ \\
Laboral de & Recuento & 25 & 3 & 28 \\
investigación & \% sobre universidad & $15,3 \%$ & $6,8 \%$ & $13,5 \%$ \\
TEU & Recuento & 26 & 7 & 33 \\
& \% sobre universidad & $16,0 \%$ & $15,9 \%$ & $15,9 \%$ \\
Laboral & Recuento & 31 & 23 & 54 \\
docente & \% sobre universidad & $19,0 \%$ & $52,3 \%$ & $26,1 \%$ \\
Universidad & Recuento & 19 & 5 & 24 \\
privada & \% sobre universidad & $11,7 \%$ & $11,4 \%$ & $11,6 \%$ \\
Total & & 163 & 44 & 207 \\
\hline
\end{tabular}

Tabla 11. Estructura del profesorado por tipo de contrato y tamaño de la universidad durante el período de estudio (2005-T12014)

\begin{tabular}{|c|c|c|c|c|c|}
\hline \multirow{2}{*}{\multicolumn{2}{|c|}{ Tipo de contrato }} & \multirow{2}{*}{\multicolumn{3}{|c|}{ Tamaño ( $n^{\circ}$ de alumnos) }} & \multirow[t]{2}{*}{ Total } \\
\hline & & & & & \\
\hline CU+TU & Recuento & $\begin{array}{r}\text { Menos } \\
\text { de } \mathbf{5 . 0 0 0} \\
0\end{array}$ & $\begin{array}{c}\text { De } 5.000 \text { a } \\
20.000 \\
41\end{array}$ & $\begin{array}{r}\begin{array}{l}\text { Más de } \\
20.000\end{array} \\
27\end{array}$ & 68 \\
\hline & \% sobre universidad &, $0 \%$ & $38,3 \%$ & $30,7 \%$ & $32,9 \%$ \\
\hline Laboral de & Recuento & 1 & 9 & 18 & 28 \\
\hline investigación & \% sobre universidad & $8,3 \%$ & $8,4 \%$ & $20,5 \%$ & $13,5 \%$ \\
\hline \multirow[t]{2}{*}{ TEU } & Recuento & 0 & 14 & 19 & 33 \\
\hline & \% sobre universidad &, $0 \%$ & $13,1 \%$ & $21,6 \%$ & $15,9 \%$ \\
\hline Laboral & Recuento & 11 & 19 & 24 & 54 \\
\hline docente & \% sobre universidad & $91,7 \%$ & $17,8 \%$ & $27,3 \%$ & $26,1 \%$ \\
\hline Universidad & Recuento & 0 & 24 & 0 & 24 \\
\hline privada & \% sobre universidad &, $0 \%$ & $22,4 \%$ &, $0 \%$ & $11,6 \%$ \\
\hline Total & & 12 & 107 & 88 & 207 \\
\hline
\end{tabular}


Tabla 12. Producción en artículos, libros y aportaciones de libros (por intervalo) por antigüedad de la universidad durante el período de estudio (2005-T12014)

\begin{tabular}{llrrr}
\hline \multirow{2}{*}{$\begin{array}{l}\text { Artículos, libros y aportaciones de } \\
\text { libros }\end{array}$} & \multicolumn{2}{c}{ Fundación de la Universidad } & \multirow{2}{*}{ Total } \\
\cline { 2 - 3 } Ninguno & Recuento & 51 & Antes de $\mathbf{1 9 8 5}$ & \\
\hline \multirow{3}{*}{ la 10} & \% sobre universidad & $31,3 \%$ & 23 & 74 \\
& Recuento & 89 & $52,3 \%$ & $35,7 \%$ \\
más de 10 & \% sobre universidad & $54,6 \%$ & 21 & 110 \\
& Recuento & 23 & $47,7 \%$ & $53,1 \%$ \\
Total & \% sobre universidad & $14,1 \%$ & 0 & 23 \\
& & 163 &, $0 \%$ & $11,1 \%$ \\
\hline
\end{tabular}

Tabla 13. Producción en artículos indexados (por intervalo) por antigüedad de la universidad durante el período de estudio (2005-T12014)

\begin{tabular}{|c|c|c|c|c|}
\hline \multicolumn{2}{|c|}{ Artículos indexados } & \multicolumn{2}{|c|}{ Fundación de la Universidad } & \multirow[t]{2}{*}{ Total } \\
\hline & & Antes de 1985 & Antes de 1985 & \\
\hline \multirow[t]{2}{*}{ Ninguno } & Recuento & 116 & 34 & 150 \\
\hline & \% sobre universidad & $71,2 \%$ & $77,3 \%$ & $72,5 \%$ \\
\hline \multirow[t]{2}{*}{1 a 5} & Recuento & 34 & 9 & 43 \\
\hline & \% sobre universidad & $20,9 \%$ & $20,5 \%$ & $20,8 \%$ \\
\hline \multirow[t]{2}{*}{ más de 5} & Recuento & 13 & 1 & 14 \\
\hline & \% sobre universidad & $8,0 \%$ & $2,3 \%$ & $6,8 \%$ \\
\hline Total & & 163 & 44 & 207 \\
\hline
\end{tabular}

Tabla 14. Producción de artículos, libros y aportaciones de libros (por intervalo) por tamaño de la universidad durante el período de estudio (2005-T12014)

\begin{tabular}{|c|c|c|c|c|c|}
\hline \multirow{2}{*}{\multicolumn{2}{|c|}{$\begin{array}{l}\text { Artículos, libros y aportaciones de } \\
\text { libros }\end{array}$}} & \multicolumn{3}{|c|}{ Tamaño ( $n^{\circ}$ de alumnos) } & \multirow[t]{2}{*}{ Total } \\
\hline & & $\begin{array}{c}\text { Menos } \\
\text { de } 5.000\end{array}$ & $\begin{array}{c}\text { De } 5.000 \mathrm{a} \\
20.000\end{array}$ & $\begin{array}{c}\text { Más de } \\
20.000\end{array}$ & \\
\hline \multirow[t]{2}{*}{ Ninguno } & Recuento & 7 & 31 & 36 & 74 \\
\hline & \% sobre universidad & $58,3 \%$ & $29,0 \%$ & $40,9 \%$ & $35,7 \%$ \\
\hline \multirow[t]{2}{*}{1 a 10} & Recuento & 5 & 64 & 41 & 110 \\
\hline & \% sobre universidad & $41,7 \%$ & $59,8 \%$ & $46,6 \%$ & $53,1 \%$ \\
\hline \multirow[t]{2}{*}{ más de 10} & Recuento & 0 & 12 & 11 & 23 \\
\hline & \% sobre universidad &, $0 \%$ & $11,2 \%$ & $12,5 \%$ & $11,1 \%$ \\
\hline Total & & 12 & 107 & 88 & 207 \\
\hline
\end{tabular}

Tabla 15. Producción de artículos indexados (por intervalo) por tamaño de la universidad durante el período de estudio (2005-T12014)

\begin{tabular}{|c|c|c|c|c|c|}
\hline \multirow{2}{*}{\multicolumn{2}{|c|}{ Artículos indexados }} & \multirow{2}{*}{\multicolumn{3}{|c|}{ Tamaño ( $n^{\circ}$ de alumnos) }} & \multirow[t]{3}{*}{ Total } \\
\hline & & & & & \\
\hline \multirow{2}{*}{ Ninguno } & Rec & 12 & 74 & 64 & \\
\hline & \% sobre universidad & $100,0 \%$ & $69,2 \%$ & $72,7 \%$ & $72,5 \%$ \\
\hline \multirow[t]{2}{*}{1 a 5} & Recuento & 0 & 27 & 16 & 43 \\
\hline & \% sobre universidad &, $0 \%$ & $25,2 \%$ & $18,2 \%$ & $20,8 \%$ \\
\hline \multirow[t]{2}{*}{ más de 5} & Recuento & 0 & 6 & 8 & 14 \\
\hline & \% sobre universidad &, $0 \%$ & $5,6 \%$ & $9,1 \%$ & $6,8 \%$ \\
\hline Total & & 12 & 107 & 88 & 207 \\
\hline
\end{tabular}




\section{DISCUSIÓN DE LOS RESULTADOS}

El objetivo de este estudio ha sido analizar la producción científica de los profesores de contabilidad de las universidades españolas. Para ello hemos tomado una muestra de 207 profesores, 169 de ellos adscritos a 7 universidades públicas y 38 a 8 privadas. Identificado el profesorado que constituye la muestra, se recopiló su producción científica (aportaciones) en el período 2005 - marzo de 2014 a partir de diversas bases de datos y buscadores de uso común.

La producción media de artículos publicados por cada profesor universitario de contabilidad de la muestra tiene una moda de valor " 0 " y una mediana de " 1 trabajo publicado en revistas". En el periodo considerado, un $45 \%$ de la muestra no parece haber publicado ningún artículo (o al menos no hemos podido identificar ninguno en nuestra búsqueda); de los restantes, un $44 \%$ ha publicado menos de un trabajo de media por año y sólo un $11 \%$ más de un trabajo de media por año.

Centrándonos en la producción más valorada en los sistemas de evaluación de méritos, los artículos en revistas indexadas en el JCR, la gran mayoría de los profesores analizados (165 de 207) no han publicado ningún trabajo en revistas JCR y 18 profesores sólo tienen 1 en todo el periodo. Únicamente un 5\% de la muestra tiene más de 5 trabajos publicados en revistas indexadas en el JCR en el período analizado (prácticamente 10 años). Respecto a artículos publicados en revistas indexadas en Scopus, el $81 \%$ de los profesores universitarios de contabilidad de la muestra no tiene ningún trabajo publicado, un 18\% de 1 a 5 trabajos y sólo un 1\% más de 5 trabajos.

Respecto al macro-área de las revistas en que se publica, entre un 50 y un $60 \%$ de los artículos escritos por profesores de contabilidad se publican en revistas no contables. Este fenómeno no se limita a los mejores trabajos, siendo aún más frecuente en la categoría de los artículos publicados en revistas "no indexadas". De los profesores de contabilidad más prolíficos, sólo un $22 \%$ publica todos sus trabajos en revistas de contabilidad mientras que un $56 \%$ publica menos de la mitad de sus artículos en revistas de contabilidad.

Estos dos resultados previos, bajo porcentaje de artículos en revistas contables y en revistas Scopus deben analizarse conjuntamente. El bajo porcentaje de artículos publicados en un conjunto de revistas sustancialmente mayor sólo puede interpretarse en términos de su baja valoración relativa, cuando la exigencia y el esfuerzo para publicar en esas revistas sea posiblemente el mismo. Así que, ante el bajo número y alta exigencia de las revistas contables JCR, parece más eficiente dirigir el esfuerzo a una revista JCR no contable que a una revista contable Scopus, cuya valoración es más baja. Inicialmente esto no es un problema si se mantiene la relevancia para el área de los resultados publicados. En la medida en que sea necesario adaptar, o dejar en un segundo plano la problemática relevante para nuestra área de conocimiento en aras de hacer más atractivo el trabajo para otra área, sí supondría un problema.

Los resultados indican que una parte importante de la producción científica (aproximadamente el $50 \%$ de los artículos publicados en revistas indexadas) de los profesores universitarios de contabilidad tienen como autores a un grupo pequeño de profesores (sobre un 5\%). En este pequeño grupo de profesores se observa que la proporción de artículos JCR que publican en revistas contables es mucho menor a la media (33 vs $51 \%$ ) y a la proporción en que ellos mismos publican en revistas contables indexadas en Scopus. Por tanto, los autores que publican más artículos de impacto lo hacen en revistas no contables en 2 de cada 3 ocasiones. Respecto a la categoría profesional de este grupo de profesores más prolíficos, la mayoría de ellos son titulares de universidad, encontrando un ayudante doctor y un contratado doctor, aparte de dos profesores de universidad privada. Este resultado invalida parcialmente la tesis de Moya et al (2015), en la medida en que no es el acceso a un puesto fijo (los TU lo son) 
lo que de alguna manera se interpreta como el fin del camino y desalienta el esfuerzo investigador. Sin embargo no se encuentra entre este grupo a ninguno que, en ese momento fuese catedrático (esto no significa que no estuviese acreditado ya). Para estos profesores, posiblemente la promoción a catedrático sea, junto con la obtención de tramos el incentivo. Por otro lado, la concentración de estos profesores en básicamente dos de las universidades de la muestra, debe ser motivo de reflexión. Es posible que haya un efecto de grupo, de especialización en un tópico y/o sea consecuencia de políticas más activas por parte de esa universidad en concreto.

No se observan diferencias significativas en la estructura de producción entre universidades públicas y privadas 3 , ni en general, ni respecto a los artículos indexados. Por el contrario, si se aprecia una diferencia significativa (al 1\%) en la estructura de la producción entre las distintas categorías de profesores, tanto en general como en los artículos indexados. De esta forma, catedráticos, titulares de universidad y contratados laborales de perfil investigador (frente a titulares de escuela universitaria y contratados laborales de perfil docente) muestran una mayor propensión a publicar. Los profesores de universidad privada muestran una posición intermedia entre estos dos grupos. La diferencia entre categorías podría explicar diferencias entre las universidades fundadas antes y después de la aprobación de la LRU, así como entre universidades pequeñas, medianas y grandes, ya que unas y otras parecen tener estructuras de personal significativamente distintas (al 0,1\%). Esta diferencia de estructura de la producción se observa en el conjunto de artículos, libros y capítulos de libros, que se muestra significativa al 5\%, pero no respecto a los artículos indexados. Por tamaño de universidad, las diferencias en la estructura de producción no son estadísticamente significativas.

La conclusión principal que se obtiene de este estudio es que en el periodo considerado hay un conjunto relativamente elevado de profesores con una producción científica baja, y que la producción de trabajos considerados "de impacto" se concentra en un número relativamente reducido de profesores. Esta baja producción se concentra, para las públicas, en las categorías laborales con perfil no investigador y en los TEU y también es muy alta en los profesores de universidades privadas.

Es preciso estudiar en detalle cuáles son las posibles causas de estos resultados. La acumulación de esa baja producción en determinadas clases de contratos puede ser indicativa de que el sistema de incentivos no funciona correctamente, de manera que la relación entre el nivel de esfuerzo necesario y la esperanza de la recompensa que pueda obtenerse hacen poco atractivo el intento. Adicionalmente, la deriva impuesta hacia un tipo de investigación específica puede tener también como efecto el abandono. Los resultados de Arquero, Jiménez-Cardoso y Laffarga (2016) también pueden aclarar algunos motivos: existe una grave disonancia entre lo que los académicos consideran relevante y útil y lo que se valora, cada vez más, en los sistemas de evaluación. La unión de dificultad de obtener resultados positivos (en cuanto a publicación y posterior valoración), la forma de evaluar (prácticamente un sí vale / no vale), la escasa cuantía de los incentivos (en comparación con actividades alternativas) y la percepción de no ser algo relevante explicaría por qué quienes no lo necesitan perentoriamente no dedican tiempo a la investigación. En este sentido,

\footnotetext{
${ }^{3}$ La inclusión de la Universidad de Navarra en la muestra puede introducir distorsiones, que hay que considerar en la interpretación de los resultados. Esta universidad es bastante mayor que la media en número de profesores y tiene entre sus filas un grupo de profesores con una producción científica muy destacable y orientada a revistas de alto impacto, lo que no es la tendencia normal en las otras privadas.
} 
realizamos de nuevo un llamamiento a considerar las especificidades de las distintas áreas a la hora de evaluar la investigación, teniendo en cuenta además que buena parte de la investigación en contabilidad debería tener un carácter marcadamente crítico-normativo y aplicado (más afines al derecho que a la economía), áreas que, de acuerdo con los resultados de Arquero et al., (2017), se están abandonando por los investigadores reconocidos.

Por último, es preciso destacar algunas dificultades que disminuyen la visibilidad, no sólo por que puedan haber influido a la baja en los niveles de producción encontrados, sino porque pueden disminuir el impacto de los trabajos. Entre éstas destacamos la dificultad de acceso a determinados datos. Especialmente los abstracts de congresos de carácter generalista que no son de acceso público o no están correctamente identificados; aunque también hay revistas no incluidas en índices, o con bloqueos de búsqueda, que son de difícil acceso a los buscadores y por tanto no proporcionan resultados o lo hacen de forma errónea.

Igualmente, es difícil identificar correctamente las autorías, por problemas de firma. El tratamiento de los apellidos españoles en BD extranjeras suele ser muy variado y también genera problemas las distintas formas de identificar la Universidad (variaciones en el nombre en español e inglés). Por último, sólo un bajo porcentaje de universidades e investigadores mantienen currículums actualizados o perfiles investigadores en la web. El uso de identificadores estandarizados (p.e. ORCID), repositorios institucionales y las campañas de unificación de firmas, pueden ayudar a solventar estos problemas en un futuro.

\section{Agradecimientos}

Este trabajo forma parte de un proyecto más amplio galardonado con el premio a Proyectos de Investigación «JOSÉ Ma FERNÁNDEZ PIRLA» en su XXI Edición (Instituto de Contabilidad y Auditoría de Cuentas y ASEPUC).

Una versión previa de este trabajo se presentó en el XVII encuentro de ASEPUC, Bilbao, 2016. Se agradecen los comentarios y sugerencias recibidas.

\section{BIBLIOGRAFÍA}

Arquero, J.L.; Jiménez Cardoso, S.M.; \& Laffarga, J. (2015) Efectos de la evaluación de la investigación en el área de Contabilidad. Trabajo presentado en XII Foro internacional sobre la evaluación de la calidad de la investigación y de la educación superior (FECIES). Sevilla.

Arquero, J. L., Cardoso, S. M. J., \& Briones, J. L. (2016). Utilidad percibida de la producción académica-contable. Opinión de los profesores universitarios y de los profesionales. Revista de Contabilidad, 19(2), 239-251.

Arquero, J. L., Jiménez-Cardoso, S. M., \& Laffarga-Briones, J. (2017). Patrones de investigación en contabilidad de los profesores con éxito académico. Spanish Journal of Finance and Accounting/Revista Española de Financiación y Contabilidad, 46(3), 327-368.

Cavero Rubio, J. A., \& Ferrández Serrano, V. (2013). El sexenio de investigación en contabilidad. EDUCADE. Revista de Educación en Contabilidad, Finanzas y Admi-nistración de Empresas, 4, 5-21. 
García Benau, M.A \& Laínez Gadea, J.A. (2004): Forever destined to be extras in a Broadway show? A discussion on the status of national accounting research in an international arena. In The real life-guide to accounting research. A behind the scene view of using qualitative research methods p. 73-94. Elsevier.

Gonzalo, J. A., \& Mora, A. (2010). Accounting research in Spain: two decades of evolution. EAA newsletter, (3), 16-18.

Jiménez-Contreras, E., de Moya Anegón, F., \& López-Cózar, E. D. (2003). The evolution of research activity in Spain: The impact of the National Commission for the Evaluation of Research Activity (CNEAI). Research policy, 32 (1), 123-142.

Moya, S., Prior, D., \& Rodríguez-Pérez, G. (2015). Performance-based incentives and the behavior of accounting academics: Responding to changes. Accounting education, 24 (3), 208-232. 